\author{
Annual scientific report \\ April 1, 2006 - March 31, 2007 \\ Fast laser excitation and ultrahigh strain-rate deformation \\ DOE DE-FG52-06NA26153 \\ Principal Investigator \\ Robert Averback \\ University of Illinois at Urbana-Champaign
}

\title{
Grant participants:
}

Graduate students: Bryan Gundrum and Samson Odunuga

Faculty: Averback, Bellon

\section{Scientific Goals:}

This research program explores the response of simple metals to ultrafast laser irradiation. Two themes are under investigation: (i) solidification of alloys under deep undercooling (ii) phase stability alloys under repeated ultrafast laser irradiation. The first area is primarily concerned with phase transitions far from equilibrium and the properties of the solid liquid interface and the supercooled liquid. The second project examines the phase evolution during laser pulsing in situations where the laser either induces local melting of the alloy (low-energy laser pulses) or plastic deformation (high-energy laser pulses).

\section{Accomplishments:}

During the first year of the grant renewal, we have made good progress toward our goals of elucidating the basic materials response to ultrafast laser irradiation. This work includes both computer simulation and experimentation, as we now describe.

(a) Application of third harmonic generation of light to monitor the thermal history of melting and solidification

With ultrafast lasers it is now possible to monitor phase transitions in real times with sub-picosecond resolution. While an important achievement in itself, it has been far more difficult to measure the thermal history of the materials as it goes through such transitions. We have a developed a method using time-resolved pump-probe 
measurements to follow the thermal history of (001) Si as it undergoes ultrafast melting and solidification. Our method employs circularly polarized, third harmonic generation (THG) of light to monitor the phase transition in time. For Si, the third order nonlinear susceptibility, $\chi^{(3)}$, has two independent terms, and the bulk polarizability can be written as

$$
P_{i}^{N L}=3 \chi_{x x y y}^{(3)} E_{i}(\vec{E} \bullet \vec{E})+\left(\chi_{x x x x}^{(3)}-3 \chi_{x x y y}^{(3)}\right) E_{i}^{3},
$$

where $\vec{E}$ is the incident electric field at the fundamental frequency. The first term is independent of crystal rotation angle for linearly polarized incident light, but it is zero for circularly polarized light. The second term is anisotropic, being a function of the angle between the polarization vector of the incident electric field and the standard crystal axis. For an isotropic medium, such as amorphous (a-) or liquid $\mathrm{Si}$, the magnitude of the optical anisotropy must be zero, and $P_{i}^{N L}=0$. We found in pump-probe experiments that following 120 fs laser pump pulses at fluences above the melt threshold, the circularly polarized THG signal decreases by over three orders of magnitude. It regains its original intensity on solidification after $\approx 2$ ns as shown in Fig. 1 . 


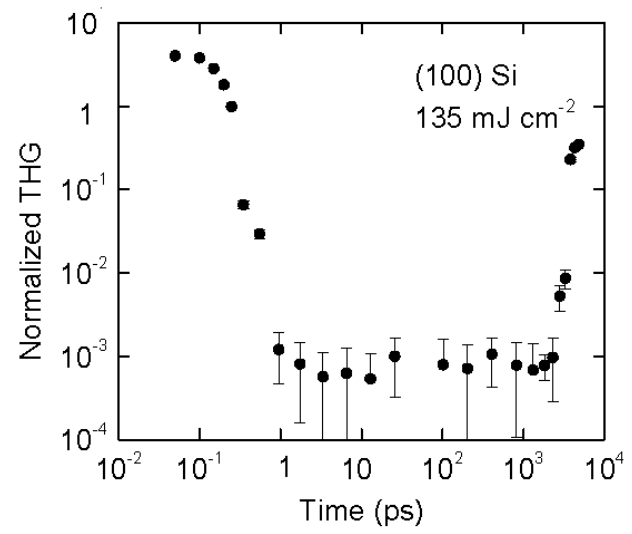

Figure 1. Normalized circularly polarized THG signal versus time for (100) Si following a 120 fs pulse of $135 \mathrm{~mJ}-\mathrm{cm}^{-2}$ laser light at $800 \mathrm{~nm}$.

We show in Fig. 2 that for Si the THG signal depends on temperature. Here it is shown that both the linear and circularly polarized signals drop markedly with increasing temperature.

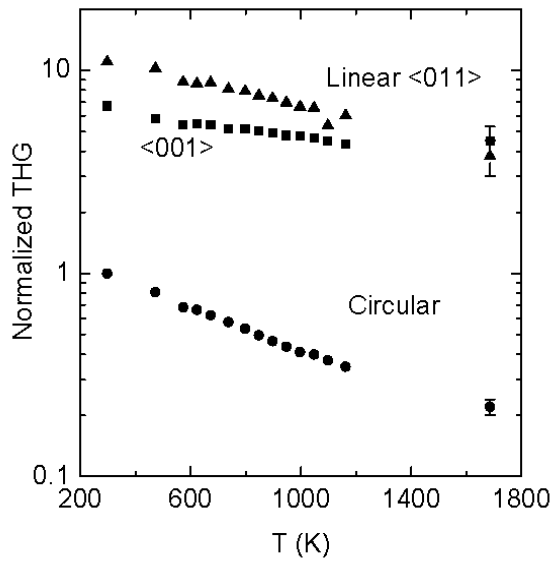

Fig. 2. Temperature dependence of THG light using $120 \mathrm{fs}$ probe pulse at $800 \mathrm{~nm}$. These are static experiments

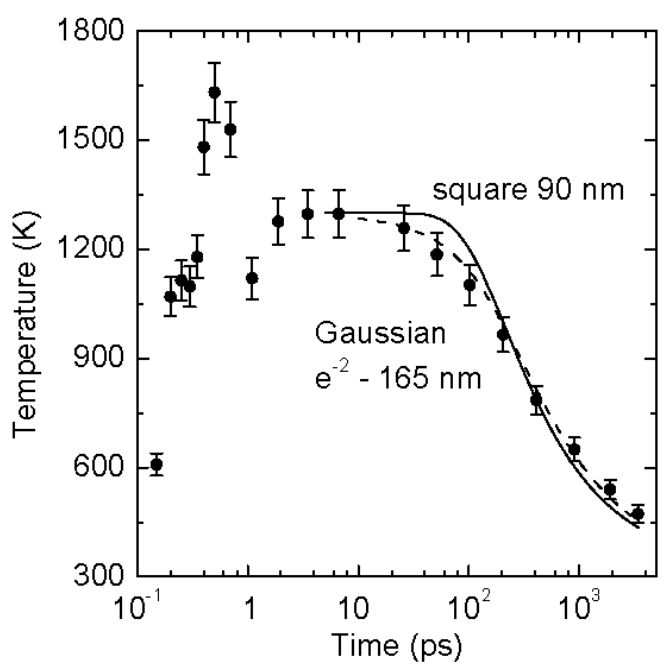

Fig. 3. Thermal history of $\mathrm{Si}(100)$ following a 120 fs pump probe at $800 \mathrm{~nm}$ that is below the melting threshold energy. The dashed and solid lines are thermal conductance model calculations. 
The data at melting temperature of Si were obtained from data like that shown in Fig. 1. In Fig.3 we show the thermal history of a sample that was irradiated with subthreshhold energy for melting. The dashed and solid line are calculated heating curves (but fitted to the initial temperature of $1250 \mathrm{~K}$ ) illustrating that the thermal history derived from the THG signal, the data points, provide an accurate picture of the melting process and with 1 ps resolution. The temperature peak at $0.5 \mathrm{ps}$ is an artifact deriving from the overlap of pump and probe beams, while the dip between 0.5 and 1.0 ps appears to signal an excitation of optical phonons modes that rapidly decays. Initial experiments on (100) $\mathrm{Cu}$ suggest that this methods can be applied to metals as well as Si. Our future work will, in fact, examine solidification in metals using both this THG methods and new measurements at LLNL using the dynamic transmission electron microscope (DTEM) in collaboration with W. King.

\section{b) Molecular dynamics simulation of shear-induced mixing}

In our previous grant funding period we had examined by molecular dynamics forced mixing in solids during high strain rate deformation [Odunuga et al. Phys. Rev. Lett. 95, 045901 (2005)]. One of the principal findings in that work is that much could be learned about the deformation mechanism by examining the relative displacements of pairs of atoms, so-called Richardson pairs in the field of turbulence, as a function of their separation. We have continued this work, in collaboration with A. Caro at LLNL, and have derived a general relationship between the coherence length of the deformation and the relative displacements of Richardson pairs, and we have applied it to case of deformation of nanocrystalline and amorphous metals (Phys. Rev. Lett, submitted). One of the interesting findings derived from this work is the importance of dislocation activity on the deformation of nanocrystalline materials. Previously it was believed that deformation in these materials was entirely due to grain boundary sliding, but we are finding that nearly half of the steady state strain derives from the dislocations. Fig. 1 
clearly shows the dislocations, both partial and perfect, in a $5 \mathrm{~nm}$ grained $\mathrm{Cu}$ sample. We are presently extending this work to learn how the coherence length of the deformation mechanisms depends grain size in metals.

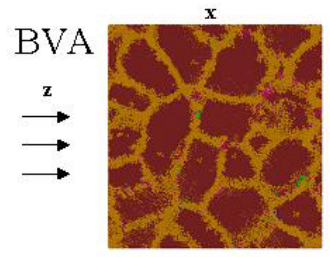

$62.5 \mathrm{ps}$

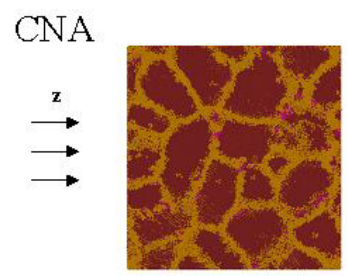

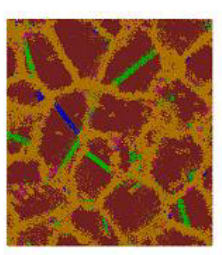

$112.5 \mathrm{ps}$

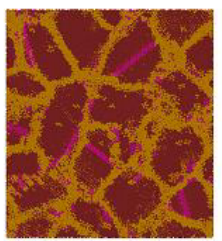

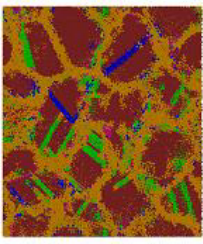

$162.5 \mathrm{ps}$

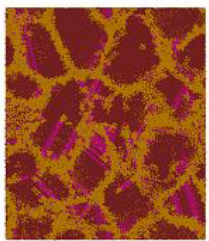

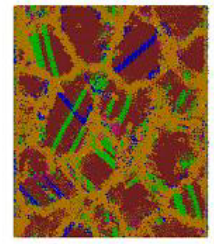

$190 \mathrm{ps}$

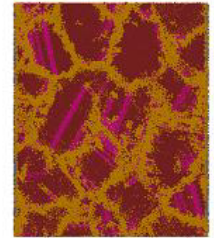

Fig. 2. Passage of dislocations through nanograins in $\mathrm{Cu}$ as a function of time during plastic deformation at a strain rate of $6 \times 10^{8}-\mathrm{s}^{-1}$. Green and blue lines in the Burgers vector analysis, BVA, represent partial and perfect dislocations, respectively. The tan lines indicate grain boundaries. The red lines in the common neighbor analysis, CAN, shows partial dislocations; this analysis does not sense perfect dislocations.

(c) Modification to work plan

There are no major changes in the program. The work plan is proceeding along the anticipated pace. One change that we do anticipate, however, is that after discussions with Dr. W King at LLNL, it appears our planned work on ultrafast electron diffraction can benefit considerably by using the DTEM instrument at LLNL. This is because the electron energy in the DTEM is $200 \mathrm{keV}$ rather than the $30 \mathrm{keV}$ planned at UIUC and therefore we can perform our experiments in transmission mode rather than the RHEED geometry. We are planning to test this possibility.

\section{Interactions with National Laboratories}

- Prof. Averback travels to LLNL on regular occasions discussing with the DTEM group (Drs. G. Campball and W. King) and Dr. H. Lorenzana, Director of a Scientific Initiative on ultrafast dynamics in shocked materials, our mutual interest in ultrafast phase transitions. 
- Prof. Averback and Prof. Bellon have been collaborating with Dr. A. Caro on MD simulations of high strain-rate deformation in nanocrysatlline materials. Prof. Averback has visited Dr. Caro at LLNL twice during the funding period.

- This summer, a new graduate student who will be joining this effort will spend the summer working with Dr. Caro at LLNL.

Publications:

Time resolved measurements of melting and solidification in Si using third harmonic generation of light

Bryan C. Gundrum, Robert S. Averback and David G. Cahill

Appl. Phys. Letts (accepted).

Crossover from superdiffusive to diffusive mixing in plastically deformed solids

P. Bellon, R.S. Averback S. Odunuga, Y. Li, P. Krasnochtchekov, A. Caro

Submitted for publication

A microscopic view of glass and liquid crystallization - revealing the structure and dynamics of liquids

Y. Ashkenazy and R.S. Averback

Submitted for publication

Presentations:

*The kinetics of crystallization: Role of point defects

TMS Annual meeting, San Antonio, March, 2006

Y. Ashkenazy and R.S. Averback,

*Kinetics of Crystallization at Extreme Undercooling: A Window into the Structure of Liquids

MRS Fall meeting (2006)

Y. Ashkenazy and R.S. Averback,

Time Resolved Measurements of Melting and Solidification in Si Using Third Harmonic Generation of Light

Frontiers in Optics 2007/Laser Science XXIII conferences in San Jose, California

Bryan C. Gundrum, Robert S. Averback, David G. Cahill

*Forced Chemical Mixing in Nanocrystalline and Amorphous Alloys subjected to Plastic Deformation

TMS Annual meeting, San Antonio, March, 2006

S. Odunuga, P. Krasnochtchekov, J. Ye, Y. Li, R. S. Averback, P. Bellon

Microstructural Evolution in Nanoscale Alloys under Plastic deformation

Fall Meeting of the MRS, November 27, 2006: Boston, Massachusetts. 